\title{
A Case of Paroxysmal Nocturnal Hemoglobinuria (PNH) in an Obstetric Patient: A South African Perspective
}

\begin{abstract}
Paroxysmal Nocturnal Hemoglobinuria (PNH) originates from an acquired genetic defect in a multipotent hematopoietic stem cell that becomes stem-cell-like in its ability to survive, expand, and self-renew. PNH is a rare condition characterized by intravascular hemolysis. PNH can arise anew or in the setting of an underlying bone marrow disorder such as aplastic anemia (AA), myelodysplastic syndrome (MDS), or primary myelofibrosis (PMF).

This case presentation documents the challenging diagnosis of PNH in the obstetric setting, in which other possible causes for a hemolytic anemia could be considered. We discuss the management of a pregnancy in the presence of PNH in a low-to-middle income setting.
\end{abstract}

Keywords: Hemoglobinuria, Paroxysmal; Anemia; Obstetrics

CMI 2019; 13(1): 1-5

https://doi.org/10.7175/cmi.v13i1.1392

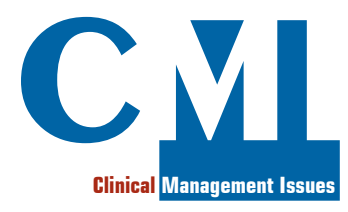

Case Report
${ }^{1}$ Department of Internal Medicine, Kalafong Tertiary Hospital, University of Pretoria, South Africa

2 Department of Internal Medicine, Steve Biko Academic Hospital, University of Pretoria, South Africa

\section{INTRODUCTION}

Paroxysmal nocturnal hemoglobinuria is a rare disease and the estimated prevalence of this disorder is in the range of one to ten cases per million population with estimates in the pregnant population being limited to case report data [1]. The median age of onset of PNH is in the mid-thirties and can affect women of childbearing age and in some instances PNH may be diagnosed in pregnancy for the first time [1,2]. There is no demonstrable ethnic or geographic distribution of the disease [1].

$\mathrm{PNH}$ represents the clonal expansion of hematopoietic stem cells that have an acquired somatic mutation in the phosphatidylinositol glycan-complementation class A (PIG-A) gene with a consequent deficiency of glycosyl phosphatidylinositol-anchored proteins including the complement-regulatory proteins CD55 and CD59. The clinical sequelae of the deficiency of these complement-regulatory proteins renders affected cells susceptible to lysis mediated via complement [3].
Laboratory investigations corroborating the diagnosis of $\mathrm{PNH}$ includes the presence of hemoglobinuria, a peripheral blood smear demonstrating red blood cell fragments and an elevated lactate dehydrogenase assay [4]. Clinical diagnosis of PNH may be confirmed with peripheral blood flow cytometry demonstrating the absence or severe deficiency of GPI-anchored proteins on at

\section{Why Do We Describe This Case}

This case highlights a rare disease with a prevalence of 1-2 cases per million people with data on the existence of this disease in the gravid population being limited to case report data. The rarity of this condition in the general population and in maternal medicine poses a dilemma for available therapeutic options and this case demonstrates the lack of availability of drugs such as eculizumab in resourcelimited countries
Corresponding author Garrick Laudin garrick.laudin@yahoo.com

Received: 25 November 2018 Accepted: 7 January 2019 Published: 16 January 2019 


\begin{tabular}{|c|c|}
\hline Vital Signs & Value \\
\hline Blood pressure (mmHg) & $104 / 76$ \\
\hline Heart rate (beats per minute) & 121 \\
\hline Respiratory rate (breaths per minute) & 13 \\
\hline Oral temperature (degrees Celsius) & 37.2 \\
\hline Oxygen saturation (pulse oximetry \%) & 95 \\
\hline Finger-prick glucose (mmol/L) & 5.3 \\
\hline
\end{tabular}

Table I. Vital signs.

least two cell lines. The detection of GPIanchored proteins may be performed by labelling cells with monoclonal antibodies or a fluorescein-tagged proaerolysin (FLAER) reagent [4]. Bone marrow biopsy is not usually indicated in most cases.

Pregnancy is generally discouraged in patients with PNH due to the high risk of both fetal and maternal mortality. Anemia in a pregnant patient with $\mathrm{PNH}$ is sometimes more severe and more frequent transfusions of packed red blood cells (PRBCs) are required [5]. Owing to the occurrence of thrombosis in up to $40 \%$ of patients with $\mathrm{PNH}$, the administration of low-molecularweight heparin (LMWH) is advised [1]. LMWH is generally administered from the time of confirmation of pregnancy to the end of the postpartum period [5].

Eculizumab is a humanized monoclonal antibody directed against the complement
C5 protein terminal, which reduces erythrocytic cellular lysis and stabilizes the plasma hemoglobin levels [6].

Eculizumab is the only drug approved for the treatment of PNH in USA, EU, and several other countries. It is currently not available in South Africa for the treatment of PNH.

\section{CASE PRESENTATION}

Miss AP was a 25-year-old female who was born in Malawi and had re-located to South Africa with her husband one year prior to consultation by the internal medicine service at Kalafong Tertiary Hospital. She was a primigravida and had a normal antenatal record except for the presence of a low hemoglobin. Other than her re-location to South Africa, our patient had no recent travel history to a malaria endemic area within South Africa.

At the time of consultation Miss AP had an estimated gestational age of 14 weeks with the initial consultation from the $\mathrm{Ob}$ stetric service being for the establishment of the route cause of Miss AP's anemia.

Miss AP had been previously diagnosed with an anemia in Malawi for which she recalls numerous admissions for blood transfusions and specifically recalls receiving transfusions of PRBCs during bouts

\begin{tabular}{|c|c|c|}
\hline Investigation & Result & Normal Range \\
\hline White cell count $\left(\times 10^{9} / L\right)$ & 2.39 & 3.9-12.6 \\
\hline Hemoglobin (g/dL) & 4.9 & $11.6-16.4$ \\
\hline Mean cell volume (fL) & 105.6 & $76-100$ \\
\hline Mean cell hemoglobin (pg) & 29.3 & $26.1-33.5$ \\
\hline Red cell distribution width (\%) & 23.1 & $12.4-17.3$ \\
\hline Platelets $\left(\times 10^{9} / L\right)$ & 249 & $186-454$ \\
\hline Smear & Scanty RBC fragments & \\
\hline C-Reactive Protein (mg/L) & 9 & $<10$ \\
\hline Total bilirubin (umo//L) & 30 & $5-21$ \\
\hline Direct/conjugated bilirubin (umo//L) & 5 & $0-3$ \\
\hline Alanine transaminase $(\mathrm{U} / \mathrm{L})$ & 14 & 7-35 \\
\hline Aspartate transaminase (U/L) & 106 & $13-35$ \\
\hline International normalized ratio (INR) & 1.01 & $\leq 1.1$ \\
\hline Fibrinogen (g/L) & 4.3 & $2-4$ \\
\hline D-dimers (mg/L) & 5.24 & $0-0.25$ \\
\hline Serum iron $(\mu \mathrm{mol} / \mathrm{L})$ & 5.0 & 8-252 \\
\hline Serum ferritin $(\mu \mathrm{g} / \mathrm{L})$ & २2 & 22-265 \\
\hline
\end{tabular}




\begin{tabular}{|l|c|c|}
\hline \multicolumn{2}{|c|}{ Investigation } & Result \\
\hline Malaria antigen/smear & Negative & \\
Anti-nuclear antibodies & Negative & \\
Extractable Nuclear Antigen & Negative & \\
Direct antibody testing (Coombs) & Negative & $208-378$ \\
Lactate dehydrogenase (U/L) & 2673 & $0.3-2.0$ \\
Haptoglobin (g/L) & 0.07 & \\
Hemoglobin electrophoresis & No abnormal hemoglobin variant \\
Glucose-6-phosphate dehydrogenase levels & Normal & $133-675$ \\
Vitamin B12 levels (pmol/L) & 484 & $7.0-45.1$ \\
Serum folate levels (nmol/L) & 55 & \\
Urine hemosiderin & Positive & \\
FLAER test and flow cytometry & PNH clone present & \\
\hline
\end{tabular}

Table III. Hemolysis specific investigations. of malaria. Limited workup for the anemia had been performed in Malawi and a tentative diagnosis of a suspected aplastic anemia was made.

Vital signs recorded in Table I revealed a resting tachycardia with other vitals being within normal limits. Urine dipsticks revealed trace proteinuria and $1+$ blood (weak positivity for blood).

General examination of our patient was unremarkable except for the presence of conjunctival rim pallor and non-pitting pedal edema.

Systems examination revealed a resting tachycardia with a left parasternal border systolic murmur. Abdominal examination revealed a palpable gravid uterus with the height of the uterine fundus at $14 \mathrm{~cm}$. There was no palpable lymphadenopathy and no hepatosplenomegaly. Examination of the musculoskeletal system did not demonstrate any discrepancy in the diameter of the calves.

Blood tests revealed low levels of: white cell count, hemoglobin, and serum iron (Table II).

Elevated levels of mean cell volume, red cell distribution width, total and direct/conjugated bilirubin, aspartate transaminase, fibrinogen, and D-dimers were also detected (Table II).

The smear showed the presence of scanty red blood cell fragments.

Bone marrow aspirate demonstrated features of a megaloblastosis which may be in keeping with chronic hemolysis. Overall bone marrow aspirate revealed marked hypercellularity with normal lymphocyte morphology. The bone marrow aspirate did not reveal any features of plasmacytosis with no other foreign cells noted.

\begin{tabular}{|c|c|}
\hline Bell Line & PNH Clone (percent \%) \\
\hline CD55- erythrocytes & 11 \\
\hline CD59- erythrocytes & 11 \\
\hline CD14 monocytes & 0.0 \\
\hline CD55- granulocytes & 71 \\
\hline
\end{tabular}

Parvovirus B19 polymerase chain reaction performed on peripheral blood was negative.

Hemolysis specific investigations detected elevated levels of lactate dehydrogenase and low levels of haptoglobin (Table III).

FLAER test and flow cytometry detected the presence of a PNH clone.

Table IV shows the PNH panel. The expression of the GPI anchored proteins, CD55 (DAF) and CD59 (MIRL) was abnormal on both neutrophils and erythrocytes. Expression of the GPI-linked proteins (CD14 on monocytes and CD16 on neutrophils) was normal. In summary, there was phenotypic evidence of PNH based on analysis of a variety of GPI-linked antibodies on red blood cells and granulocytes.

A diagnosis of classical PNH was made after systematically excluding other probable causes of a process of intravascular hemolysis. Miss AP was referred from her antenatal clinic at Kalafong Tertiary Hospital to the Obstetric service at Steve Biko Academic Hospital. She was jointly managed by $\mathrm{Ob}-$ stetric Medicine, Maternal Fetal Medicine as well as the Hematology Service. She declined termination of pregnancy after careful counselling about a guarded prognosis. Miss AP was commenced on enoxaparin (LMWH) from time of diagnosis (16 weeks)
Table IV. PNH panel

Laboratory Service-

NHLS, South Africa).
(National Health 
until the birth of her infant at 37 weeks. As the post-natal period in $\mathrm{PNH}$ is associated with a high incidence of thrombosis, she was placed on warfarin for 3 months postdelivery. Miss AP was supported during her pregnancy with folic acid and other hematinics, as well as transfusions of packed red blood cells when her hemoglobin dropped below $7 \mathrm{~g} / \mathrm{dL}$. Miss AP had an uneventful pregnancy with no episodes of thrombosis and delivered a live male baby via spontaneous vaginal delivery at 37 weeks.

\section{DISCUSSION}

PNH or Paroxysmal Nocturnal Hemoglobinuria is a rare disease entity which has a prevalence of 1-2 cases per million people [1]. The disease has a slight female preponderance and women of child-bearing potential are also affected [2]. The exact incidence of $\mathrm{PNH}$ in pregnancy is unknown and only case report data on $\mathrm{PNH}$ in pregnancy exists.

$\mathrm{PNH}$ is a non-neoplastic human disease caused by a somatic mutation of the X-linked phosphatidylinositol glycancomplementation class A gene in hematopoietic stem cells which makes red blood cells more vulnerable to lysis mediated by complement $[1,2]$.

The long-term complications of $\mathrm{PNH}$ include a chronic intravascular hemolysis complicated by anemia, venous thromboembolism as well as bone marrow failure $[1,4]$.

The clinical diagnosis of $\mathrm{PNH}$ in the setting of an obstetric patient can be a diagnostic challenge as the range of signs and symptoms present can be confounded with various pregnancy complications like preeclampsia, HELLP syndrome or pregnancy-associated thrombocytopenia. It is particularly important to try and distinguish a HELLP syndrome from a PNH crisis as the two entities may show overlap in symptomatology (i.e. nausea, abdominal discomfort) and abnormal laboratory findings of intravascular hemolysis (elevated lactate dehydrogenase, elevated unconjugated bilirubin, low haptoglobin, and low platelet count) [2].

$\mathrm{PNH}$ in pregnancy is associated with increased risks of complications such as thromboembolic diseases (e.g. Budd-Chiari syndrome), hypertensive disorders like preeclampsia, and cerebrovascular diseases. $\mathrm{PNH}$ in pregnancy can cause significant fetomaternal morbidity and mortality with the estimated maternal mortality ranging from $5.8 \%$ to $20.8 \%$. More than $45 \%$ of pregnancies in women with $\mathrm{PNH}$ result in either spontaneous miscarriage or termination. Of the women who give birth, more than half deliver prematurely, an event that can have negative implications for the health of the new-born baby $[3,5]$.

Eculizumab is a humanized monoclonal antibody that binds to the complement protein $\mathrm{C} 5$ and blocks terminal complement activation. During pregnancy eculizumab has shown a low rate of maternal complications up to now. However, expertise in managing pregnant patients with $\mathrm{PNH}$ with eculizumab is limited. In fact, prospective trials are unlikely to be initiated, due to the rarity of the disease. The drug is still listed in pregnancy as a category C drug ("animal reproduction studies have shown an adverse effect on the fetus and there are no adequate and well-controlled studies in humans, but potential benefits may warrant use of the drug in pregnant women despite potential risks" [7]), but potential benefits may outweigh potential risks [5]. The cost of eculizumab, trade name Soliris ${ }^{\circledR}$, is approximately 18,000 USD per dose, making it one of the most expensive drugs in the United States [8]. The exorbitant cost of the drug makes its use in $\mathrm{PNH}$ in resource-constrained countries almost unjustified.

Key Points

- PNH is a rare acquired disease with an estimated incidence of 1 to 5 cases per million individuals

- The exact incidence of PNH in pregnancy is unknown as only case report data on the condition exist

- PNH results in intravascular hemolysis and other conditions in pregnancy causing hemolysis also require consideration as diagnoses

- Thrombosis is one of the most feared complications of PNH in pregnancy as it can result in fetal loss as well as significant maternal morbidity/mortality

- Standard of care for patients in resource-limited settings is mainly supportive with the use of hematinics and with the appropriate transfusion of blood products 


\section{Funding}

This article has been published without the support of sponsors.

Conflicts of Interests

The authors declare they have no competing financial interests concerning the topics of this article.

\section{REFERENCES}

1. Brodsky RA.Pathogenesis of paroxysmal nocturnal hemoglobinuria. 2017. Available at https:// www.uptodate.com/contents/pathogenesis-of-paroxysmal-nocturnal-hemoglobinuria (last accessed January 2019)

2. Lauritsch-Hernandez LS, Kraehenmann F, Balabanov S, et al. Eculizumab application during pregnancy in a patient with paroxysmal nocturnal hemoglobinuria: A case report with review of the literature. Clin Case Rep 2018; 6: 1582-7; https://doi.org/10.1002/ccr3.1634

3. Miyasaka N, Miura O, Kawaguchi T, et al. Pregnancy outcomes of patients with paroxysmal nocturnal hemoglobinuria treated with eculizumab: a Japanese experience and updated review. Int J Hematol 2016; 103: 703-12; https://doi.org/10.1007/s12185-016-1946-x

4. Hill A, DeZern AE, Kinoshita T, et al. Paroxysmal nocturnal haemoglobinuria. Nat Rev Dis Primers 2017; 3: 17028; https://doi.org/10.1038/nrdp.2017.28

5. Kelly RJ, Höchsmann B, Szer J, et al. Eculizumab in pregnant patients with Paroxysmal Nocturnal Hemoglobinuria. N Engl J Med 2015; 373: 1032-9; https://doi.org/10.1056/ NEJMoa1502950

6. Bastos JMC, Pinheiro PL, Rocha LC, et al. Therapeutic challenges in pregnant women with paroxysmal nocturnal hemoglobinuria: A case report. Medicine (Baltimore) 2018; 97: e12155; https://doi.org/10.1097/MD.0000000000012155

7. AA.VV.FDA Pregnancy Categories. Available at https://www.drugs.com/pregnancy-categories. html (last accessed January 2019)

8. Dinerstein C. Why Is Soliris the Most Expensive Drug In The US? American Council in Science and Health. May 27, 2017. Available at https://www.acsh.org/news/2017/05/27/why-solirismost-expensive-drug-us-11333 (last accessed January 2019) 